\title{
Urban Greening Using an Intelligent Multi-Objective Location Modelling with Real Barriers: Towards a Sustainable City Planning
}

\author{
Meher Nigar Neema ${ }^{1 *}$, Khandoker Md. Maniruzzaman ${ }^{2}$, Akira Ohgai $^{3}$ \\ ${ }^{1}$ Department of Urban and Regional Planning, Bangladesh University of \\ Engineering and Technology, Dhaka, Bangladesh \\ ${ }^{2}$ Department of Urban and Regional Planning, College of Architecture Planning, \\ University of Dammam, Dammam, KSA \\ ${ }^{3}$ Department of Architecture and Civil Engineering, Toyohashi University of Technology, Toyohashi, Japan \\ Email: "mnnneema@yahoo.com
}

Received May $22^{\text {nd }}$, 2013; revised June $28^{\text {th }}$, 2013; accepted July $15^{\text {th }}$, 2013

Copyright (c) 2013 Meher Nigar Neema et al. This is an open access article distributed under the Creative Commons Attribution License, which permits unrestricted use, distribution, and reproduction in any medium, provided the original work is properly cited.

\begin{abstract}
Greenery is one of the important ingredients for urban planning with a sustainable environment. Increasing parks and open spaces (POS) to offer a greater diversity of green spaces have substantial impact on its environment in many mega cities around the world. However, any place cannot be a potential site for POS due to multi-objective modeling nature of POS planning. In this paper, an intelligent multi-objective continuous optimization model is thus developed for locating POS with particular emphasis on greeneries that will potentially benefit and facilitate the planning of a sustainable city. Three environmentally incommensurable factors analyzed with the help of geographic information systems (GIS) namely air-quality, noise-level, and population-distribution have been considered in the model and a genetic algorithm (GA) is used to solve the continuous optimization problem heuristically. The model has been applied to Dhaka city as a case study to find the optimal locations of additional POS to make it a sustainable city by ameliorating its degraded environment. The multiple objectives are combined into a single one by employing a dynamic weighting scheme and a set of non-dominated Pareto optimal solutions is derived. The obtained alternative non-dominated solutions from the robust modelling approach can serve as a candidate pool for the city planners in decision making for POS planning by selecting an alternative solution which is best suited for the prevailing land-use pattern in a city. The model has successfully demonstrated to provide optimal locations of new POS. In addition, we found that locations of POS can be optimized even by integrating it with land cover and uses like lakes, streams, trails (for simplicity which were considered as a barrier constraint in the model) to rejuvenate added beauties in a city. The obtained results thus indicate that the developed multi-objective POS location model can serve as an effective tool for urban POS planning maintaining sustainable environment.
\end{abstract}

Keywords: Urban Greening; Parks and Open Space; Sustainable Environment; GA and GIS; Intelligent Location Modelling

\section{Introduction}

In an integral urban planning, greeneries can predominantly be found in parks, playgrounds, gardens etc. Parks and open spaces (POS) i.e. greeneries in particular can substantially improve the livability of land uses and city environment. Its important functions are: 1) potential amelioration of microclimate, 2) absorbtion of pollutants, 3) reduction of noise levels including a significant improvement of the lifestyle of city-dwellers by allowing admixture with nature and promoting social interactions (Schipperijn et al., 2010; Szeremeta et al., 2009; Gobster, 1998; Morancho, 2003; Uy \& Nakagoshi, 2008; Chiesura, 2004; Egger, 2006; Kong et al., 2010; Borrego et al., 2006; Lam, 2005; Poggio \& Vrcaj, 2009; Nordh et al., 2009; BenDor et al., 2013; Choumert, 2010; Neema et al., 2013; Neema \&

\footnotetext{
"Corresponding author.
}

Ohgai, 2013; Neema et al., 2008). Importantly, the necessity of POS in landscape planning and design can be realized in densely populated cities around the globe (Lam et al., 2005).

As such a densely populated city, Dhaka has emerged as a fast growing mega-city. In 1975, it started with a population of only 2.2 million which has culminated to 16 million in 2010. In 2015, a predicted population is 21 million. It can be envisaged that due to this rapid growth of population in Dhaka, it is confronted with a big challenge to deal with serious environmental degradation due mainly to the significantly diminished number of POS. Once, Dhaka city was considered as a green city but now it has left with only $21.6 \%$ open space (but not green spaces) of its total area with huge population (SDNP, 2005). Statistically, it can be calculated that only approximately 8 sq. meter POS is available per person in Dhaka city (Uddin, 2005), which is rather insufficient for a healthy sustainable city. In 
contrary, POS for greeneries in built up areas of a city like Dhaka is ought to be considered as one of the most valuable, protective and attractive elements. Surprisingly, due to inadequate planning there are insufficient and non-optimal locations of POS in the city. Therefore, an efficient POS planning is indispensable for attaining a sustainable environment in Dhaka city.

However, an intelligent POS planning generally include multiple criteria so that it could stimulate optimum benefits in urban environment. Thus, the planning for POS locations can be considered as a multi-objective facility location optimization problem. The relevant objective criteria for the optimal location of additional POSs (for greeneries) mainly are: population distribution, air quality, noise level, and physical barriers. With the considered physical barriers we mean industrial areas, existing parks, lakes, big rivers, airport zones, highways and mountain ranges. Some barriers might hinder POS planning in its interior but some barriers can be used with POS in an integrated way.

Interestingly, many researchers focused on applying operations research models in ecological reserves which considered also wetlands and water bodies (McDonnell et al., 2002; Camm et al., 2002; Drechsler \& Wtzold, 2001). But no systematic research has been conducted yet to develop and apply operations research models on POS planning for city greening including barrier concept.

In our previous research (Neema \& Ohgai, 2010), we developed a genetic algorithm (GA)-based multi-objective location model for open spaces without considering any barriers. In this paper, we extend the model to include barriers to develop a robust intelligent model for finding optimal locations of POS using our GA-based multi-objective continuous optimization scheme. In this research, we consider POSs particularly for urban greeneries. These POSs include city parks, local parks, playgrounds, neighbourhood open spaces and other green areas. The model thus developed is applied to Dhaka city as a case study.

The paper is organized in the following way. In the next section, we illustrate our intelligent multi-objective model formulation with barriers. Then, we explain the calculation of shortest permitted distance with barrier constraints. After that we briefly describe our algorithms. Then, we apply the model thus develop to Dhaka city (as a case study) for providing more POS. Next, we provide computational results and discussion. Finally, we provide some concluding remarks.

\section{Formulation of Intelligent Multi-Objective POS Model with Barriers}

Like most real-world planning problems, urban parks and open spaces planning are ill-structured containing important factors that are difficult to quantify and represent precisely. This type of planning problem often contains more than one objective and decision makers are often required to evaluate solutions according to multiple criteria and their preferences (Zhang \& Armstrong, 2008). The solution to such problems requires simultaneous optimization of multiple, often competing criteria (or objectives), is usually computed by combining them into a single criterion to be optimized, according to some utility function. In many cases, however, the utility function is not well known prior to the optimization process. The whole problem should then be treated as a multi-objective problem. In this way, a number of solutions (Pareto-optimal) can be found which provide decision makers with insight into the character- istics of the problem before a final solution is chosen (Fonseca \& Fleming, 1991). A detail formulation for a multi-objective continuous optimization model for parks and open space (POS) is given below. First, we need to define the objectives of the model and then explain elaborately the adopted concept in the inclusion of barriers in the model which is the main focus of this study.

\section{Problem Definition and Model Objectives}

The multiple objectives of the model are to locate POS by minimizing distances from: populated areas $\left(f_{1}\right)$, air-polluted areas $\left(f_{2}\right)$, and noisy areas $\left(f_{3}\right)$. For locating POS, we define our problem space as a 2-D continuous rectangular region, $\xi^{2}$ with known maximum and minimum $x, y$ coordinates. In $\xi^{2}$, demands for facilities (POS in our model), $u_{i}$ are distributed over a set of given points $u_{j}$ (demand points) with assigned positive weights $w_{j}$ (population, air quality and noise level in our model). In $\xi^{2}$, we denote barrier regions for locating facilities (i.e. POS) by $B_{k}, k=1,2, \cdots, q$. A multi-objective function is set to determine the approximate optimal locations of the facilities without placing in barrier regions as well as minimizing total travel distance with respect to each measure. The multiple objectives are represented by following functions:

- Minimizing population weighted distance

$$
\min f_{1}=\sum_{i=1}^{m} \sum_{j=1}^{n}\left\langle P_{w_{j} d}\left(u_{i}, u_{j}\right)\right\rangle \cdot P_{c_{i j}}
$$

- Minimizing air quality weighted distance

$$
\min f_{2}=\sum_{i=1}^{m} \sum_{j=1}^{n}\left\langle A Q_{w_{j} d}\left(u_{i}, u_{j}\right)\right\rangle \cdot A Q_{c_{i j}}
$$

- Minimizing noise level weighted distance

$$
\min f_{3}=\sum_{i=1}^{m} \sum_{j=1}^{n}\left\langle N L_{w_{j} d}\left(u_{i}, u_{j}\right)\right\rangle \cdot N L_{c_{i j}}
$$

where, $u_{i}$ denotes the location of facility (where $i$ ranges from 1 to $m$ ), $u_{j}$ the location of a demand point (where $j$ ranges from 1 to $n) . P, A Q, N L$ respectively stand for population, air quality and noise level. $P_{w_{i} d}, A Q_{w_{j} d}$ and $N L_{w_{i} d}$ are respective weights of demand point $u_{j}$ for population, air quality and noise level. The allocation decision variables for weighted distances of population, air quality and noise level are given respectively by $P_{c_{i j}}, A Q_{c_{i j}}$ and $N L_{c_{i j}} . d\left(u_{j}, u_{i}\right)$ is the travel distance between two points $u_{i}$ and $u_{j}$.

We combine all three single objective functions into a composite function, $F$. The multi-objective function takes the following form:

$$
\min F=\left\{f_{1}, f_{2}, f_{3}\right\}
$$

We apply a weighting scheme to obtain the composite function $F$. Using the scheme in our GA-based model, we generate a different random weight vector, $v$ for each solution (chromosome) where $v=\left[w_{1}, w_{2} \text { and } w_{3}\right]^{T}$ (i.e. consists of three weights denoted by $w_{1}, w_{2}$ and $w_{3}$ respectively for three objectives i.e. $f_{1}, f_{2}$ and $f_{3}$ ). We then multiply each objective function by the corresponding weight and aggregate to obtain the composite objective function, $F$. We generate three random numbers between 0 and 1 , denoted by $r_{1}, r_{2}$ and $r_{3}$ to derive $w_{1}$, $w_{2}$ and $w_{3}$ respectively. We denote $T$ for transpose. 


$$
\begin{gathered}
\min F=f_{1} w_{1}+f_{2} w_{2}+f_{3} w_{3} \\
v=\left[\begin{array}{lll}
w_{1} & w_{2} w_{3}
\end{array}\right]^{T} \\
w_{1}=\frac{r_{1}}{r_{1}+r_{2}+r_{3}} \\
w_{2}=\frac{r_{2}}{r_{1}+r_{2}+r_{3}} \\
w_{3}=\frac{r_{3}}{r_{1}+r_{2}+r_{3}}
\end{gathered}
$$

The assumed constraints for the model are:

- We prevent siting of facilities inside barrier regions.

- If facility $u_{i}$ is allocated to demand point $u_{j}$, i.e., where the population weighted distance, $P_{w_{d}}\left(u_{j}, u_{i}\right)$ is at minimum between demand point $u_{j}$ and facility $u_{i}$, then $P_{c_{i j}}=1$; otherwise it is 0 . Similarly, we derive the allocation decision variables for air quality and noise level.

- The distance between two points $u_{j}$ and $u_{i}$ is Euclidean distance. We discuss in the next section how to calculate the distance in presence of barriers.

- The total weight for all objectives is equal to 1 .

The mathematical representation of the constraints are as follows:

$$
\left.\begin{array}{l}
u_{i} \notin B_{k}, \\
\sum_{i=1}^{m} P_{c_{i j}}=1, P_{c_{i j}}=0 \text { or } 1, \\
\sum_{i=1}^{m} A Q_{c_{i j}}=1, A Q_{c_{i j}}=0 \text { or } 1, \\
\sum_{i=1}^{m} N L_{c_{i j}}=1, N L_{c_{i j}}=0 \text { or } 1, \\
d\left(u_{i}, u_{j}\right)=\left\{\left|x_{i}-x_{j}\right|^{2}+\left|y_{i}-y_{j}\right|^{2}\right\}^{1 / 2}, \\
w_{1}+w_{2}+w_{3}=1,
\end{array}\right\}
$$

Next, we describe the procedure in details to include real barriers in the model.

\section{Inclusion of Barriers in the Model}

The barriers $B_{k}, k=1,2, \cdots, q$ consist of multiple circular barriers $\left(B_{c}=1,2, \cdots, c\right)$ and line barriers $\left(B_{l}=1,2, \cdots, l\right)$ such that $q=c+l$. We assume non-elongated shaped barriers such as industrial areas, airport zones, existing parks, water bodies etc. as circular barriers, $B^{c}$ and elongated shaped barriers such as lakes, rivers, highways, borders etc. as line barriers, $B_{l}$. We further subdivide $B^{c}$ in two categories i.e. flexible barriers, $\left(F L B_{c}\right)$ and fixed barriers, $\left(F L B_{c}\right)$. We define a $F L B_{c}$ as the region i.e. a water body where location is not feasible but travel through the water body may be possible using a boat and a $F L B_{c}$ as the region i.e. industrial plant, existing parks etc. where neither travel nor location is allowed. We further consider a line barrier, $B_{l}$ as the region (such as a lake) where location is not feasible but in real world, one might expect to have some exit points, $e p_{\rho}$ (e.g. over bridge) for travel through such elongated shaped barriers.

We adopt the following assumptions for barrier inclusion in the model:
1) Barrier representation: Each circular barrier, $B_{c}$ is defined by a circle. The area of the $B_{c}$ is the equivalent area of the existing real barrier. The centroid of existing real barrier is used to draw each $B_{c}$. Each line barrier, $B_{l}$ is defined by a line and it is the center line of an existing real barrier. The exit points, $e p_{\rho}$ on a line barrier, $B_{l}$ are determined from the location of exit points on the existing real barrier.

2) Facility location: Facility location is not allowed inside a $B_{c}$. A buffered distance is used for all $B_{l}$ to define the prohibited region of facility location. The prohibited region from $B_{l}$ is denoted by $B_{l\{\emptyset\}}$.

3) Travel through the barriers: It is permitted to travel through flexible barriers $F L B_{c}$ and the distance between two points $u_{j}, u_{i} \notin B_{k}$ in $\xi^{2}$ that are separated by a $F L B_{c}$ is measured in Euclidean metric, $d\left(u_{j}, u_{i}\right)$. It is not permitted to travel through fixed barriers $F L B_{c}$ but possible to travel along the boundary of $F L B_{c}$. For line barriers $B_{l}$, it is permitted to travel only through the defined exit points $e p_{\rho}$. The shortest permitted distance between two points $u_{j}, u_{i} \notin B_{k}$ in $\xi^{2}$ which are separated by a $F L B_{c}$ and/or a $B_{l}$ is denoted by $\tilde{d}\left(u_{i}, u_{j}\right)>$ $d\left(u_{j}, u_{i}\right)$. Shown in Figure 1 is a pictorial depiction of the considered problem space with travel distance between facilities and demand points through barriers.

In the following section, we describe in details the calculation of shortest permitted distance, $\tilde{d}\left(u_{i}, u_{j}\right)$ in presence of fixed barriers and/or line barriers to incorporate into the model.

\section{Calculation of Shortest Permitted Distance}

There are three subsections in this section. We present the calculation of shortest permitted distance between facilities and demand points in presence of fixed barriers, in presence of line barriers and in presence of both fixed and line barriers.

\section{In Presence of Fixed Barriers}

We assume that a facility point, $u_{i}$ and a demand point, $u_{j}$ which are not inside a barrier, $B_{k}$ (i.e. $u_{j}, u_{i} \notin B_{k}$ ) visible when the straight-line joining the points does not intercept a fixed barrier $F I B_{c}$. Similarly, the set of facility points $u_{i}$ that are invisible from a demand point $u_{j}$ are in the shadow region of $u_{j}$ (see in Figure 2(a)).

If two tangents are drawn from point $u_{j}$ to $F I B_{c}$, two points of tangency can be obtained: right, $t_{r}\left(u_{j}\right)$ and left, $t_{l}\left(u_{j}\right)$. The conventions for "right" and "left" were adopted based on the bisector that starts from $u_{j}$ and passes through the center of $F I B_{c}$ following (Klamroth, 2004). Similarly, two points of tangency i.e $t_{r}\left(u_{j}\right)$ and $t_{l}\left(u_{j}\right)$ can be found by drawing two tangents from $u_{i}$ to $F I B_{c}$. There are two permitted paths between $u_{i}$ and $u_{j}$ :

$\tilde{d}_{1}\left(u_{j}, u_{i}\right)$-a permitted-path constructed with the points of tangency $t_{l}\left(u_{j}\right)$ and $t_{r}\left(u_{j}\right)$ (see in Figure 2(b)).

$\tilde{d}_{2}\left(u_{i}, u_{j}\right)$ - a permitted-path through the points of tangency $t_{r}\left(u_{j}\right)$ and $t_{l}\left(u_{j}\right)$.

Following the technique adopted by Katz and Cooper (Katz \& Cooper, 1981) we calculated the permitted-path length as:

$$
\begin{aligned}
\tilde{d}_{1}= & d\left(u_{j}, t_{l}\left(u_{j}\right)\right)+2 r \arcsin \left[\frac{d\left(t_{l}\left(u_{j}\right), t_{r}\left(u_{i}\right)\right)}{2 r}\right] \\
& +d\left(t_{r}\left(u_{i}\right), u_{i}\right) \\
= & d\left(u_{j}, t_{l}\left(u_{j}\right)\right)+r \alpha+d\left(t_{r}\left(u_{i}\right), u_{i}\right)
\end{aligned}
$$




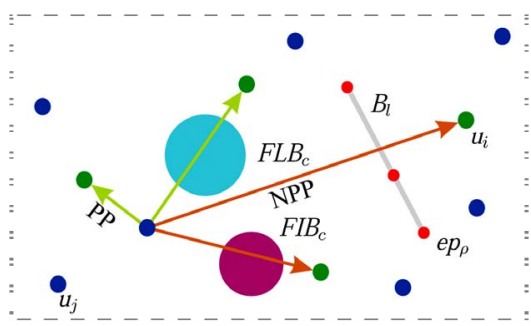

Figure 1.

A schematic of a problem space and travel distance between facilities and demand points in presence of barriers: $u_{j}=$ demand point, $u_{i}=$ facility point, $F L B_{c}=$ flexible barrier, $F I B_{c}=$ fixed barrier, $B_{1}=$ line barrier, $e p_{\rho}=$ exit points of line barrier, NPP = non-permitted path, PP $=$ permitted path.

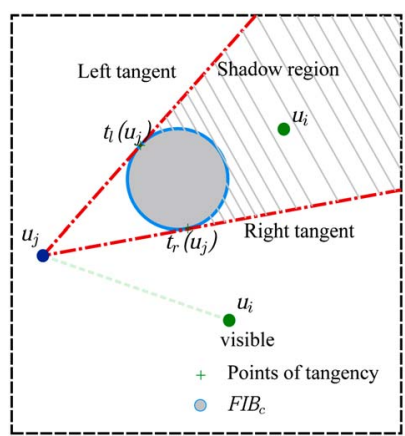

(a)

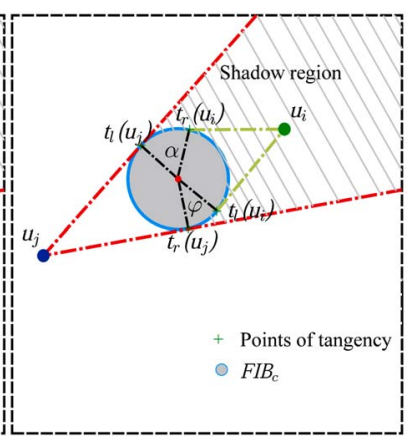

(b)
Figure 2.

(a) The shadow region of a demand point $u_{j}$ (b) Permitted path between a facility point $u_{i}$ and a demand point $u_{j}$.

$$
\begin{aligned}
\tilde{d}_{2}= & d\left(u_{j}, t_{r}\left(u_{j}\right)\right)+2 r \arcsin \left[\frac{d t_{r}\left(u_{j}\right), t_{l}\left(u_{i}\right)}{2 r}\right] \\
& +d\left(t_{l}\left(u_{i}\right), u_{i}\right) \\
= & d\left(u_{j}, t_{r}\left(u_{j}\right)\right)+r \phi+d\left(t_{l}\left(u_{i}\right), u_{i}\right)
\end{aligned}
$$

where, $r$ signifies the radius of $F I B_{c} . \alpha$ and $\varphi$ are the angles between the radii to $t_{l}\left(u_{j}\right)$ and $t_{r}\left(u_{i}\right)$, and $t_{r}\left(u_{j}\right)$ and $t_{l}\left(u_{i}\right)$ respectively. The shortest one between these two paths is considered as the shortest permitted path.

\section{In Presence of Line Barriers}

Here, we assume the two points $u_{j}, u_{i} \notin B_{k}$ are separated by a line barrier, $B_{l}$ if they are on opposite side of $B_{l}$. If a $B_{l}$ is a straight line, the two end points of $B_{l}$ are used to derive whether the positions of $u_{i}$ and $u_{j}$ are on the same side or on the opposite side of $B_{l}$. If a $B_{l}$ is a curve line, some vertex points are used to define the $B_{l}$ in the model. The vertices are used for the procedure of deriving whether positions of $u_{i}$ and $u_{j}$ are on the same side or on the opposite side of $B_{l}$. First, the distances from $u_{i}$ and $u_{j}$ to each vertex are calculated. Then the straight lines between the nearest two vertices of $u_{i}$ and that of $u_{j}$ are used to derive whether the positions of $u_{i}$ and $u_{j}$ are on the same side or on the opposite side of $B_{l}$. If there is a $B_{l}$ between $u_{i}$ and $u_{j}$, the travel distance from $u_{i}$ to $u_{j}$ is permitted only through some $e p_{\rho}$ of $B_{l}$. In such a situation, to calculate the shortest permitted distance from $u_{i}$ to $u_{j}$, the following procedure is used:

Suppose, there are four exit points $e p_{\rho}$ ( $\rho: 1$ to 4$)$ present in $B_{l}$ (see in Figure 3). At first, a straight line is drawn that starts at $u_{j}$ and ends at $u_{i}$. This straight line intersects $B_{l}$ at a point intp $p_{\gamma}$, here $\gamma=1$. Next, the distances from intp $p_{\gamma}$ to all $e p_{\rho}$ are calculated and denoted by $d_{1}, d_{2}, d_{3}$ and $d_{4}$. The shortest one among these four distances is selected. In this illustration, $d_{2}$ is the shortest, so $e p_{2}$ is the nearest exit point from intp $p_{\gamma}$. The nearest exit point is denoted by $e p_{\rho}^{*}$. So, the shortest permitted travel distance from $u_{j}$ to $u_{i}$ is the sum of the distances from $u_{j}$ to $e p_{\rho}^{*}$ and from $e p_{\rho}^{*}$ to $u_{i}$ and the equation is given as below:

$$
\tilde{d}\left(u_{i}, u_{j}\right)=d\left(u_{j}, e p_{\rho}^{*}\right)+d\left(e p_{\rho}^{*}, u_{i}\right)
$$

\section{In Presence of Fixed and Line Barriers}

In this section, we assume there are a fixed barrier $F I B_{c}$ and a line barriers $B_{l}$ exist in between a demand point $u_{j}$ and a facility point $u_{i}$ (see in Figure 4). First, tangents are drawn from both $u_{j}$ and $u_{i}$ to $F I B_{c}$ (similar to the treatment presented in subsection 3.1). Then we obtain four tangent points i.e. $t_{l}\left(u_{j}\right), t_{r}\left(u_{j}\right), t_{l}\left(u_{i}\right)$ and $t_{r}\left(u_{i}\right)$. The tangents from $u_{i}$ to $F I B_{c}$ intersects a $B_{l}$. Two points of intersection are found as $i n t p_{1}$ and $i n t p_{2}$. The nearest exit points from $i n t p_{1}$ and $i n t p_{2}$ are derived using the technique described. The nearest exit points from intp $p_{1}$ and int $p_{2}$ respectively are $e p_{2}$ (denoted by $e p_{\rho}^{* l}$ ) and $e p_{3}$ (denoted by $\left.e p_{\rho}^{* r}\right)$. The two shortest permitted distances are shown in Equations (14) and (15). The shortest one between $d_{1}$ and $d_{2}$ is selected as the shortest permitted path.

$$
\begin{aligned}
\tilde{d}_{1}= & d\left(u_{j}, t_{r}\left(u_{j}\right)\right)+\operatorname{arc}\left(t_{r}\left(u_{j}\right), t_{l}\left(u_{i}\right)\right) \\
& +d\left(t_{l}\left(u_{i}\right), e p_{\rho}^{* l}\right)+d\left(e p_{\rho}^{* l}, u_{i}\right) \\
\tilde{d}_{2}= & d\left(u_{j}, t_{l}\left(u_{j}\right)\right)+\operatorname{arc}\left(t_{l}\left(u_{j}\right), t_{r}\left(u_{i}\right)\right) \\
& +d\left(t_{r}\left(u_{i}\right), e p_{\rho}^{*_{r}}\right)+d\left(e p_{\rho}^{*_{r}}, u_{i}\right)
\end{aligned}
$$

Next, we describe about the genetic algorithms to formulate the multi-objective POS location model to include the barriers.

\section{Genetic Algorithm for the Multi-Objective POS Model with Barriers}

In this section, we present briefly our GA-based model where we mainly focus on the algorithmic steps used for the inclusion of barriers. The flowchart of our GA-based model with barriers is presented in Figure 5. Details on genetic algorithms can be found in (Neema et al., 2011). In our GA, each chromosome (i.e. individual) corresponds to a potential solution.

In the initialization process, a population of solutions i.e. chromosomes is created randomly. The number of solutions (population size) in a population is predetermined. Then the solutions are checked for whether the random locations of facilities are inside or outside the barrier regions. Following steps were adopted to prevent siting of a facility inside a barrier region:

Step 1: Generate random locations of facilities $u_{i}$ in $\xi^{2}$.

Step 2: Check facility $u_{i} \notin B_{k}$.

Step 3: If $u_{i} \in B_{k}$, relocate the $u_{i}$. The relocation process is shown in Figure 6.

Step 4: If $u_{i} \in B_{c}$, draw a straight line starting from the 


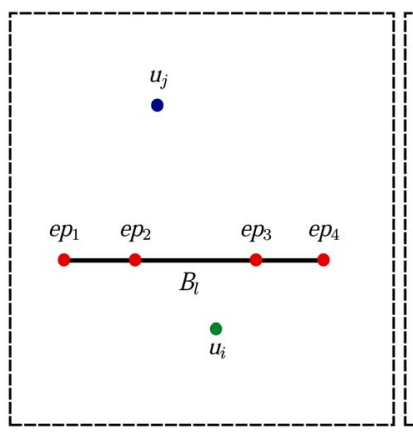

(a)

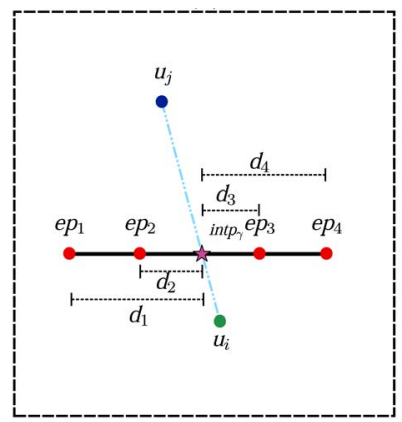

(c)

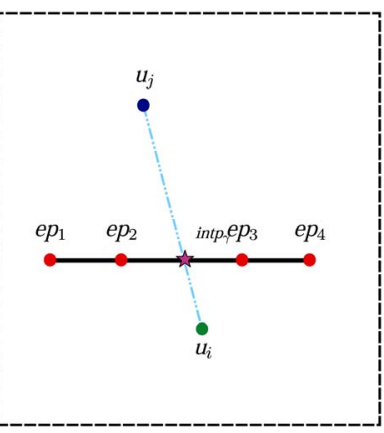

(b)

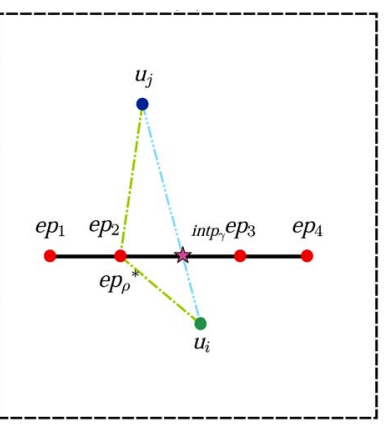

(d)
Figure 3.

The shortest permitted distance from $u_{j}$ and $u_{i}$ : (a) a $B_{1}$ with four $e p_{\rho}$, (b) the intersection point intp $p_{\gamma}$, (c) distances from intp $p_{\gamma}$ to all $e p_{\rho}$, and (d) the shortest permitted distance.

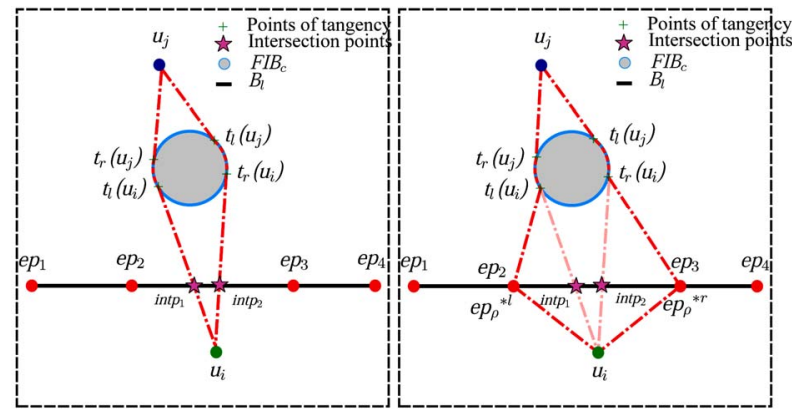

Figure 4.

(a) Presence of fixed and line barrier between a demand point and a facility (b) Permitted path in presence of fixed and line barrier.

center of $B_{c}$, that passes through the $u_{i} \in B_{c}$, and intersects the boundary of $B^{c}$ at a point $u_{i}^{*} \notin B_{c} . u_{i}^{*}$ is the nearest feasible location of the $u_{i} \in B_{c}$. Move the $u_{i} \in B_{c}$ to $u_{i}^{*} \notin B_{c}$.

The objective functions are utilized in the process of evaluating each solution.

In this step, for the distance measure the following steps are followed:

Step 1: If $u_{i} \in B_{l\left\{\xi_{1}\right.}$ of a $B_{l}$, calculate the distances from $u_{i} \in B_{l\{\rho\}}$ to all $e p_{\rho}$ of the $B_{l}$. Draw a straight line from the nearest $e p_{\rho}$ to $u_{i} \in B_{l\left\{\delta_{0}\right\}}$ and extend so that it intersects the boundary of $B_{l\{\oint\}}$. Move $u_{i} \in B_{l\{\rho\}}$ to the intersection point. The intersection point is the new location of $u_{i} \notin B_{l\{\wp\}}$ and is denoted by $u_{i}^{*} \notin B_{l\{\{\}}$.

Step 2: Check whether there is a $B_{k}$ in between $u_{j}$ and $u_{i} \notin B_{k}$.

Step 3: If there is no $B_{k}$ or only a $F I B_{c}$ exists in between $u_{j}$

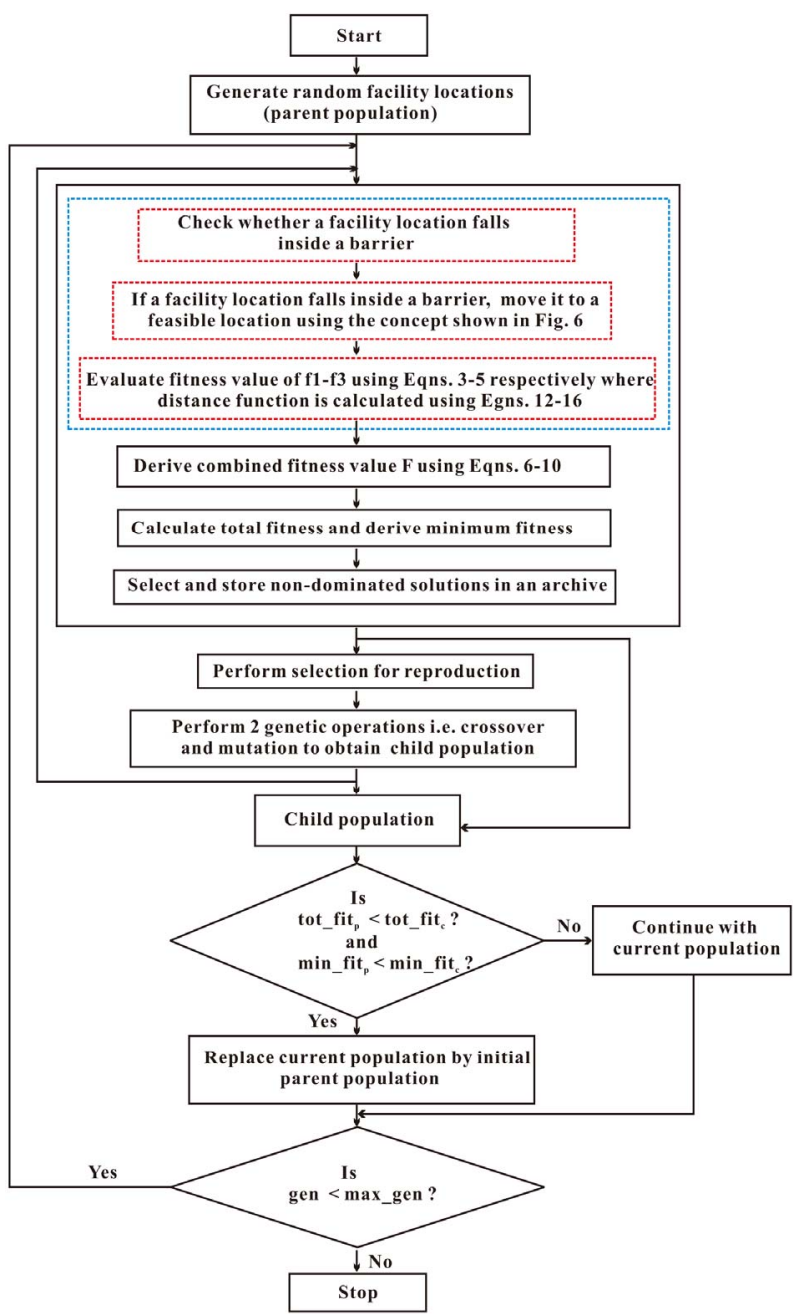

Figure 5.

Flow chart showing the genetic algorithm of multi-objective facility (POS) location problem with barriers.

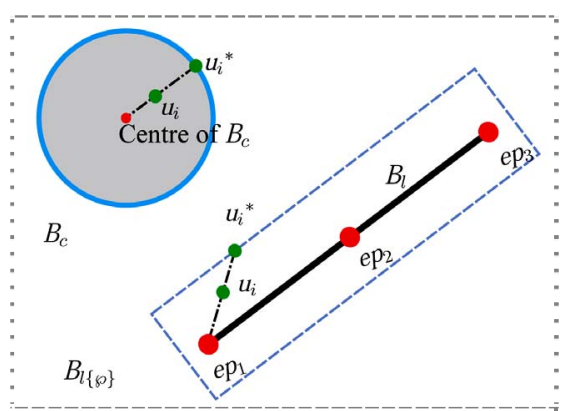

Figure 6.

Relocation of a facility to a feasible location.

and $u_{i}$, the distance between $u_{j}$ and $u_{i}$ is unconstrained. Calculate all the unconstrained distances between $u_{i}$ and $u_{j}$ in $\xi^{2}$ using Euclidean metric, $d\left(u_{j}, u_{i}\right)$.

Step 4: Calculate the distances between $u_{j}$ and $u_{i}$ in $\xi^{2}$ that are separated by a $F I B_{c}$ using Equations (11) and (12).

Step 5: Calculate the distances between $u_{j}$ and $u_{i}$ in $\xi^{2}$ that are separated by a $B_{l}$ using Equation (13).

Step 6: Calculate the distances between $u_{j}$ and $u_{i}$ in $\xi^{2}$ that 
are separated by both of $F I B_{c}$ and $B_{l}$ using Equations (14) and (15).

The non-dominated solutions are sorted from the obtained solutions to store it in an archive. The selection process for reproduction saves the better solutions. Therefore, the stronger (having better fitness) chromosomes survive, while weaker chromosomes die out. We follow roulette wheel selection method. For the reproduction process, genetic operations are performed in the parent chromosomes. This will generate offspring chromosomes. In our GA, a single-point crossover operation is carried out, where two parent chromosomes interchange their genetic material (bits) after a randomly decided single point. The mutation operation is performed to bring diversity of the solutions in the offspring population. Mutation is a random change of one or more bits. In the reproduction process to perform the next generation, we choose the better one between the parent and the offspring population. The total fitness and the minimum fitness of both population are compared for this selection.

Figure 5 shows the GA process used for multi-objective POS location problem with barriers. The GA process continues until the prefixed number of generations has been reached. After finishing the GA cycle, final sorting of non-dominated solutions is performed among the solutions stored in the archive.

Next, we present a case study applying the multi-objective model with barriers to find the approximate optimal locations of some new POS in Dhaka city.

\section{A Case Study on Dhaka City}

In our previous research, we showed 0.22 acres per 1000 people open space is available in Dhaka city. This is far below the recommended standards in different countries in the world (Neema \& Ohgai, 2010). As noted parks and green areas in Dhaka city have been diminished significantly during the last four decades. The continuous growth of population is presumebly the underlying mainstay of such depletion. In contrary, a well distributed optimized green space is regarded as one of the main ingredients of an environmentally friendly city. The optimal locations were obtained simultaneously optimizing three multiple objectives i.e. POS near: a) populated areas, b) air quality degraded area, and c) noise pollution areas including a constraint (barrier).

The prime objective of this case study is to locate some new parks and open spaces in Dhaka city. For modelling purpose, we assume there are some demand generating points in the problem space Dhaka. The centroids of city wards (a total of 90 wards) are considered as the demand points whose spatial coordinates and demands are known. As the model is formulated with continuous optimization scheme, any place could be a potential site for a POS. But the problem is that there are various constraints in reality especially many existing barriers of different types and sizes. Therefore, we need to incorporate these barrier constraints into the model to avoid unrealistic POS planning in any of such barriers. The required input data for the model was prepared employing ArcGIS 9.1 software. The following procedures and consideration were adopted:

1) We confined the problem space with the bounding rectangle of the city.

2) The centroids of 90 wards of the city is considered as the demand generating points. Three levels of demand (i.e. population, air quality, noise level) of each ward are assigned as the weights to each demand point. Details can be found elsewhere (Neema \& Ohgai, 2010).

3) We consider the existing parks and open space, industrial areas and market areas of the city as fixed barriers $F I B_{c}$, the water bodies of the city as flexible barriers $F L B_{c}$ and the lakes of the city as line barriers $B_{l}$. The total number of $F I B_{c}, F I B_{c}$ and $B_{l}$ are respectively 219, 119 and 6. We exclude the rivers from our barrier considerations as it passes mostly through the outside of the ward areas. To simply the model, we merge some small existing barriers.

4) We set the numbers of new POS in the problem space to be 30 , each of which contains 50 acres of area. Details of these considerations can be found in ((Neema \& Ohgai, 2010) where we estimated that the city needs 1505 acres of area for additional POS. For the sake of simplification of simulation, we assume the size of all new POS is to be equal.

Now, we represent the spatial distribution of existing barriers and different levels of demand for providing more POS. It can be observed that there exist different types and sizes of physical barriers in Dhaka, shown in Figure 7(a). There are a big Industrial region, some existing POS and a lake in the central part, a large existing POS in the northern part, some small industrial regions in the southern part, some lakes in the eastern part, and market areas and small size water bodies throughout the city. Among these barrier regions, we represent the elongated shaped barriers using line shapes $B_{l}$ whose exit points are denoted by $e p_{\rho}$ and non-elongated shapes using circular shapes $B_{c}$. We included the barriers in the coding of the model to restrict placing new POS locations within these regions.

The calculated ward-wise population distribution (required for objective function $f_{1}$ ) is presented in Figure 7(b). Specifically many highly populated areas are devoid of sufficient numbers of POS. POSs are mostly concentrated in a few places and extensive areas are lack of it.

Depicted in Figure 7(c) is the ward-wise air quality distribution (required for objective function $f_{2}$ ) in the city. For air quality data we considered the concentration of $\mathrm{SO}_{2}$ in the air. An area is considered to be polluted when the average $\mathrm{SO}_{2}$ concentration is above $40 \mathrm{ppb}$ level.

Evidently, there are significant spatial variations and extremely high concentrations of $\mathrm{SO}_{2}$ in the central and the southeastern industrial-zone of the city. The maximum level of $\mathrm{SO}_{2}$ is $100 \mathrm{ppb}$ which also agrees well with a previous report (Azad \& Kitada, 1998). Reportedly, the air-pollution enclaves northwest to south-east regions including the regions that fall alongside the river (Buriganga).

Basically, Dhaka being the capital city and the hub of commercial activity, the air-pollution problem of it is more acute. The air quality of the city is being badly degraded day by day.

Noise level distribution (required for objective function $f_{3}$ ) of the city is presented in Figure 7(d). As expected, the areas of the city with existing POS and lakes have less noise pollution. It reveals that POS and lakes do have a significant impact on reducing noise level. So, noise level is considered as another objective function in the model to reduce noise level of the city by providing more POS (i.e. green areas) near noise polluted areas.

Next, we present modeling results to show the effective implementation of the multi-objective model with barriers.

\section{Results}

The genetic algorithm (GA) of the model was coded in C++ 


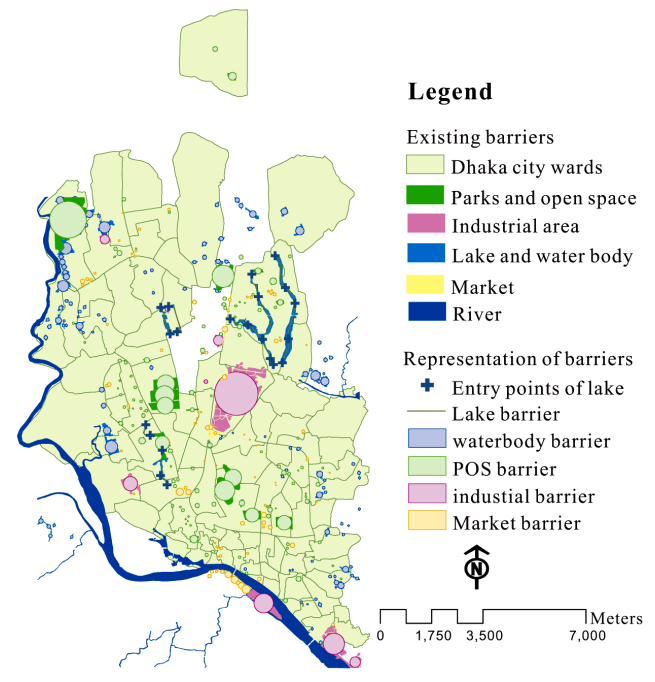

(a)

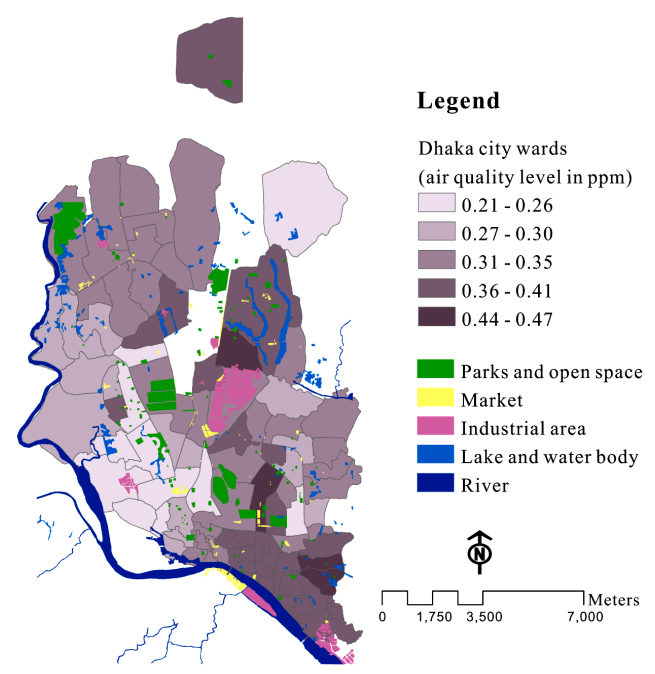

(c)

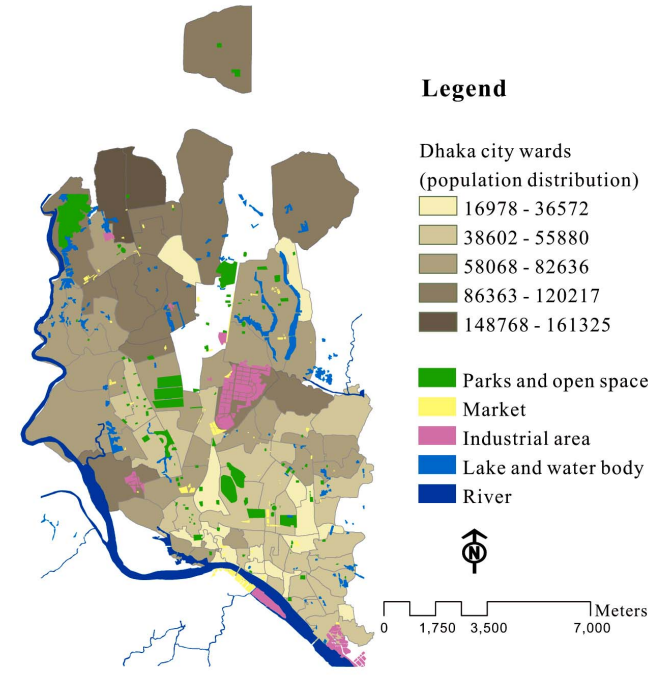

(b)

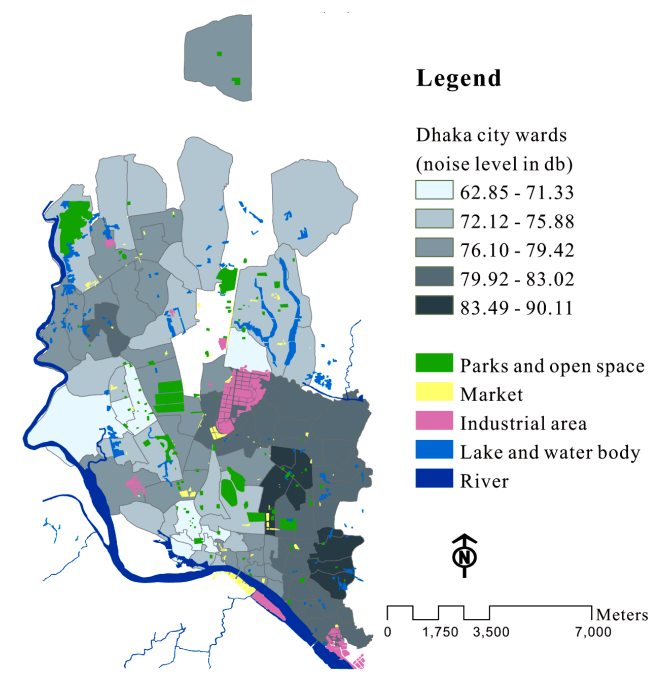

(d)

Figure 7.

Ward wise distribution of: (a) barriers, (b) population (c) air quality and (d) noise level.

programming language. The parameters of GA were set as follows: a population size of 100 , a crossover rate of 0.25 , a mutation rate of 0.009 and the maximum generation of 100 . The model parameters have empirically been shown to give better results.

Using different rand seed generators ten independent simulations were conducted.

Multi-objective optimization does not restrict to find a unique single solution of a given problem for multiple objectives optimization. Instead, it generates a pool of non-dominated Pareto optimal solutions. Therefore, first we evaluated the Pareto-optimality of the model. The non-dominated Paretooptimal solutions obtained from all iterations of each run are presented in Table 1. This table shows solutions from three objectives, compromise solutions and associated weight vectors.

Table 2 depicts the statistics of the results of Table 1. The table shows that the sum of the mean value of the non-dominated compromise solutions of three objectives is minimum in run 3 . So, we considered the results obtained from run 3 as the best.

Next, we derive non-dominated solutions of each iteration from run 3. The results are plotted in Figure 8 to delineate the Pareto front (i.e. trade off surface). City planners can use these solutions as a candidate pool for decision making. Figure 8(a) shows all non-dominated compromise solutions. The lower bound of non-dominated compromise solutions is presented as a close-up view in Figure 8(b).

From the alternative solutions presented in Table 1, the decision makers may choose a desirable weight vector based on existing barriers and objective factors. For the POS location planning in Dhaka city, we selected three weight vectors (shown in red italic font) from the obtained results of Table 1 to derive minimum weighted distances and minimum POS location points.

The underlying reasons to select such weight vectors are to investigate the effects on POS locations with real world barriers if more priority is given to population or air quality or noise 
Table 1.

Non-dominated solutions.

\begin{tabular}{|c|c|c|c|c|c|c|c|c|c|c|}
\hline \multirow[t]{2}{*}{ Run } & \multirow[t]{2}{*}{ Soln } & \multicolumn{3}{|c|}{ Solutions from three objectives } & \multicolumn{3}{|c|}{ Compromise solutions from three objectives } & \multicolumn{3}{|c|}{ Weight vectors } \\
\hline & & $f_{1}$ & $f_{2}$ & $f_{3}$ & $f_{1} w_{1}$ & $f_{2} w_{2}$ & $f_{3} w_{3}$ & $w_{1}$ & $w_{2}$ & $w_{3}$ \\
\hline \multirow{3}{*}{1} & 1 & 6887143.44 & 8469454.06 & 8452588.54 & 151391.00 & 8132368.38 & 150612.32 & 0.02 & 0.96 & 0.02 \\
\hline & 2 & 11842043.58 & 13434643.41 & 13422661.71 & 11371606.23 & 316951.25 & 216560.45 & 0.96 & 0.02 & 0.02 \\
\hline & 3 & 6909749.51 & 8504027.03 & 8496442.61 & 2863896.52 & 3246983.42 & 1730818.95 & 0.41 & 0.38 & 0.20 \\
\hline \multirow{4}{*}{2} & 1 & 7076954.88 & 8662485.56 & 8663683.67 & 18817.68 & 16584.21 & 8624060.36 & 0.00 & 0.00 & 1.00 \\
\hline & 2 & 7014950.12 & 8591020.86 & 8587052.83 & 62189.27 & 103579.68 & 8407394.63 & 0.01 & 0.01 & 0.98 \\
\hline & 3 & 7038883.26 & 8621309.42 & 8615972.75 & 62827.83 & 91546.68 & 8447578.09 & 0.01 & 0.01 & 0.98 \\
\hline & 4 & 7056233.26 & 8636960.17 & 8623082.33 & 237441.24 & 6839020.09 & 1504885.67 & 0.03 & 0.79 & 0.17 \\
\hline \multirow{2}{*}{3} & 1 & 260121.49 & 238324.36 & 234548.99 & 242128.17 & 8537.66 & 7821.98 & 0.93 & 0.04 & 0.03 \\
\hline & 2 & 252678.84 & 230654.18 & 233047.35 & 36937.53 & 171125.71 & 26078.39 & 0.15 & 0.74 & 0.11 \\
\hline \multirow{2}{*}{4} & 1 & 6946966.18 & 8574622.74 & 8556127.92 & 110812.71 & 14455.98 & 8405222.28 & 0.02 & 0.00 & 0.98 \\
\hline & 2 & 11885792.30 & 13500370.53 & 13486573.06 & 11217015.78 & 418665.31 & 340610.03 & 0.94 & 0.03 & 0.03 \\
\hline \multirow{3}{*}{5} & 1 & 6830041.59 & 7723299.86 & 7728392.79 & 19086.23 & 7505877.22 & 195969.39 & 0.00 & 0.97 & 0.03 \\
\hline & 2 & 11657667.16 & 13242989.67 & 13242422.38 & 23588.97 & 12946438.17 & 269743.12 & 0.00 & 0.98 & 0.02 \\
\hline & 3 & 6824074.53 & 7719164.83 & 7717566.85 & 2147330.01 & 1913605.10 & 3375872.85 & 0.31 & 0.25 & 0.44 \\
\hline \multirow{3}{*}{6} & 1 & 6990346.96 & 8569324.44 & 8566024.27 & 47486.08 & 7153635.50 & 1356953.93 & 0.01 & 0.83 & 0.16 \\
\hline & 2 & 6926747.86 & 8524046.41 & 8516424.34 & 2067824.33 & 3727936.95 & 2249434.47 & 0.30 & 0.44 & 0.26 \\
\hline & 3 & 6953959.36 & 8561869.73 & 8541657.30 & 2725823.70 & 2221344.63 & 2977384.73 & 0.39 & 0.26 & 0.35 \\
\hline \multirow{4}{*}{7} & 1 & 10223703.84 & 13466379.77 & 11818530.02 & 111727.45 & 28756.24 & 11664136.46 & 0.01 & 0.00 & 0.99 \\
\hline & 2 & 7001226.37 & 8557396.71 & 8556553.17 & 6720984.04 & 30709.77 & 311791.59 & 0.96 & 0.00 & 0.04 \\
\hline & 3 & 6922733.62 & 8522394.43 & 8516101.67 & 6861294.59 & 52277.80 & 23340.92 & 0.99 & 0.01 & 0.00 \\
\hline & 4 & 6945123.53 & 8544967.36 & 8531930.04 & 360008.84 & 7350838.03 & 750044.55 & 0.05 & 0.86 & 0.09 \\
\hline \multirow{2}{*}{8} & 1 & 7020172.51 & 8597104.03 & 8591444.32 & 692299.02 & 18403.96 & 7725801.44 & 0.10 & 0.00 & 0.90 \\
\hline & 2 & 7016226.55 & 8591482.48 & 8571351.86 & 1933874.32 & 2700387.75 & 3514779.67 & 0.28 & 0.31 & 0.41 \\
\hline \multirow{4}{*}{9} & 1 & 11901581.45 & 13501056.83 & 13488040.56 & 11730930.92 & 142255.32 & 51279.75 & 0.99 & 0.01 & 0.00 \\
\hline & 2 & 11860811.64 & 13478017.09 & 13470729.03 & 65762.92 & 13014960.46 & 388117.04 & 0.01 & 0.97 & 0.03 \\
\hline & 3 & 11846746.97 & 13462899.30 & 13456584.37 & 10536650.74 & 712658.72 & 775798.89 & 0.89 & 0.05 & 0.06 \\
\hline & 4 & 15133743.99 & 18387667.69 & 20027924.57 & 887261.51 & 1219814.70 & 17525099.65 & 0.06 & 0.07 & 0.88 \\
\hline \multirow{3}{*}{10} & 1 & 271047.44 & 227856.62 & 217396.43 & 3360.09 & 2474.95 & 212340.10 & 0.01 & 0.01 & 0.98 \\
\hline & 2 & 287367.02 & 248577.00 & 237935.77 & 257234.78 & 23157.82 & 2782.60 & 0.90 & 0.09 & 0.01 \\
\hline & 3 & 268483.57 & 222464.32 & 217592.01 & 165940.79 & 34645.75 & 49218.64 & 0.62 & 0.16 & 0.23 \\
\hline
\end{tabular}

level. The adopted criteria for these selections include: 1) all the three objectives are important for POS planning in the problem space, the weight of each objective should not less than $20 \%$ of total weight and 2) more priority will be given to one objective with respect to others.

The model was executed three times by fixing each weight vector in each run to find a minimum solution. It can be observed that minimum solution with iterations does not alter after 93, 27 and 43 iterations by using the weight vectors $v_{1}, v_{2}$ and $v_{3}$ respectively. So, the minimum solution obtained using each weight vector after 100 iterations is taken as the optimum solution.

The distribution of new 30 POS locations with barriers employing a weight vector $v_{1}$ or $v_{2}$ or $v_{3}$ was plotted in GIS environment and is shown in Figures 9(a)-(c). New POS locations are marked with red color and ward numbers (with black color).

\section{Discussion}

From the developed multi-objective continuous optimization model for open spaces in urban planning, one can find that not a single sitting of open spaces fall within barriers interior that 
Table 2.

Non-dominated solutions.

\begin{tabular}{cccccccccccc}
\hline & 1 & 2 & 3 & 4 & 5 & 6 & 7 & 8 & 9 & 10 & Avg \\
\hline$\varepsilon_{a}$ & \multicolumn{1}{c}{10} & & & & & & & \\
\hline$\varepsilon_{1}$ & 4795631.25 & 95319.01 & 139532.85 & 5663914.25 & 730001.74 & 1613711.37 & 3513503.73 & 1313086.67 & 5805151.52 & 142178.56 & 2381203.09 \\
$\varepsilon_{2}$ & 3898767.68 & 1762682.67 & 89831.69 & 216560.65 & 7455306.83 & 4367639.02 & 1865645.46 & 1359395.86 & 3772422.30 & 20092.84 & 2480834.50 \\
$\varepsilon_{3}$ & 699330.57 & 6745979.69 & 16950.19 & 4372916.16 & 1280528.45 & 2194591.04 & 3187328.38 & 5620290.55 & 4685073.83 & 88113.78 & 2889110.26 \\
\hline Sum $^{\mathrm{b}}$ & 9393729.50 & 8603981.36 & $\mathbf{2 4 6 3 1 4 . 7 2}$ & 10253391.05 & 9465837.02 & 8175941.44 & 8566477.57 & 8292773.08 & 14262647.66 & 250385.18 & 7751147.86 \\
Min $^{\mathrm{c}}$ & 7841698.88 & 8573163.59 & 234141.63 & 8530490.98 & 7436807.97 & 7924553.05 & 6936913.31 & 8149041.74 & 11924466.00 & $\mathbf{2 1 8 1 7 5 . 1 4}$ & 6776945.23 \\
Min-max $^{\mathrm{d}}$ & 3246983.42 & 8407394.63 & $\mathbf{1 7 1 1 2 5 . 7 1}$ & 8405222.28 & 3375872.85 & 2977384.73 & 6861294.59 & 3514779.67 & 11730930.92 & 212340.10 & 4890332.89 \\
Num $^{\mathrm{e}}$ & 3 & 4 & 2 & 2 & 3 & 3 & 4 & 2 & 4 & 3 & 3 \\
\hline
\end{tabular}

${ }^{\mathrm{a}}$ Mean value of all non-dominated compromise solutions after a run; ${ }^{\mathrm{b}}$ Sum of the mean values of the three objectives; ${ }^{\mathrm{c}}$ Minimum non-dominated aggregated compromise solutions; ${ }^{\mathrm{d}}$ Maximum of the minimum non-dominated aggregated compromise solutions; ${ }^{\mathrm{e}}$ Total number of non-dominated solutions in each run.

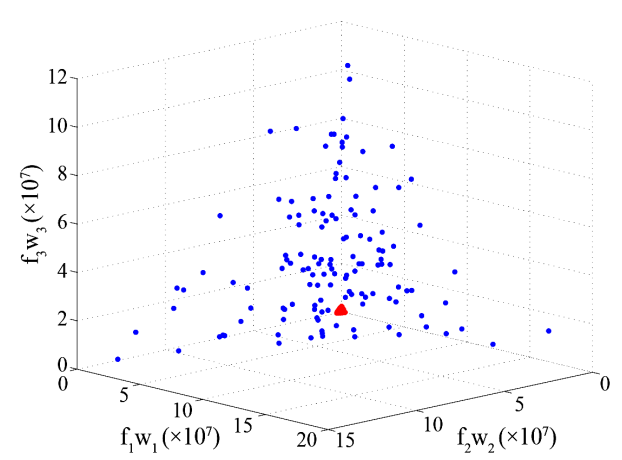

(a)

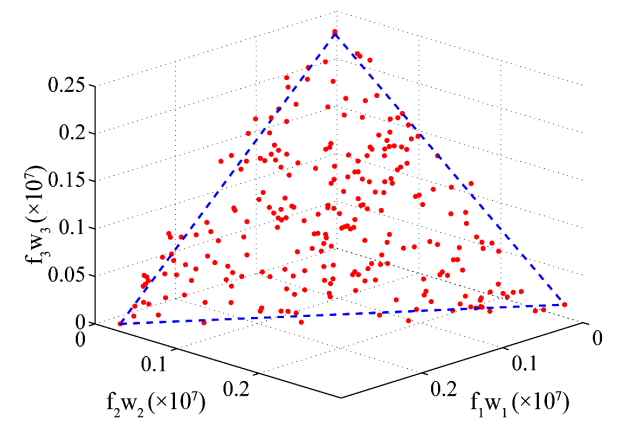

(b)

Figure 8.

Pareto-front (trade-off surface) of non-dominated compromise solutions from all iterations of run 3: (a) all solutions and (b) solutions in lower bound.

demonstrates the successful implementation of the model.

With assigned higher priority to degraded air quality, a significant number of new locations of open spaces are found within the wards 60 - 80 (see in Figure 9(b)). The distribution of locations of most of the open spaces in these areas (with no large barriers) can be attributed to those wards which have degraded air quality and possess moderately high population. However, some locations of open spaces deviated from the expected results: the locations marked " 6 ", “9”, “15", and " 27 " distributed near existing open spaces and the locations marked "0", “4”, “8”, “10”, “21" located near lake type barrier and water-body type barrier. From urban planning point of view these locations can also be justifiable to obtain an ideal ambience for a beautiful urban planning to rejuvenate city dwellers.

Evidently, when a higher priority was given to degraded sound quality, a significant number of locations of new open spaces were found preferably in the noisy wards as expected (see in Figure 9(c)). However, there are some exceptions: five locations of open spaces (marked “6”, “9”, “15”, “22”, and "27”) are found near existing open spaces. The model also sitted five locations of open spaces marked "0", “4”, "8”, “19" and " 25 " near the lake type barrier. These results could be due to the combined effects of the degraded condition of sound quality (south-east part and center of the city) and air quality (east and south-east).

With an exception from the modelling results, a few number of open spaces were found to be located on the peripheries of circular barriers. Using the weight vector, $v_{1}=\left[\begin{array}{llll}0.41 & 0.38 & 0.02\end{array}\right]^{T}$ two locations of open spaces marked " 1 " and "4" have shown to fall on the peripheries (see in Figure 9(a)).

Moreover, it can be observed that there are five new locations of open spaces marked " 1 ”, “18”, “23”, “26” and “28” near existing open spaces and five other locations marked " 0 ", "5”, “7”, " 14 " and "19" are found near a lake. These results are expected because we are optimizing locations of open spaces using multiple objectives including barriers. We used continuous optimization scheme where locations of open spaces can be anywhere in the space based on weighted combined effects of air- and sound-quality, population density. Barriers are just used as constraints to optimize locations of open spaces in the model. In addition, no buffer region was considered for circular barriers during the optimization process. However, from the urban planning point of view such locations thus obtained can be accepted based on the fact that areas near to lakes are devoid of any open spaces and commercial areas (where air quality is in worst condition) have insufficient existing open spaces. The practical consideration for such locations would be that city planners can change the type of open spaces (for example, locate playground and/or neighborhood open spaces near an existing city park) thus obtained from simulation for locations of new open spaces. The locations of open spaces near lakes and water bodies, can effectively be planned in an integrated way (i.e. open spaces and lakes) by the city planners. This could bring a beautiful image and a better environment to rejuvenate city dwellers. However, details of different types of open 


\section{Legend}

Dhaka city wards

\ New 30 POS locations by $\mathrm{v} 1$

Parks and open space

Parks and open space barrier

Industrial area

industial barrier

Market

Market barrie

Lake and water body

lake barrier

$+\quad$ entry points of lake

waterbody barrier

River

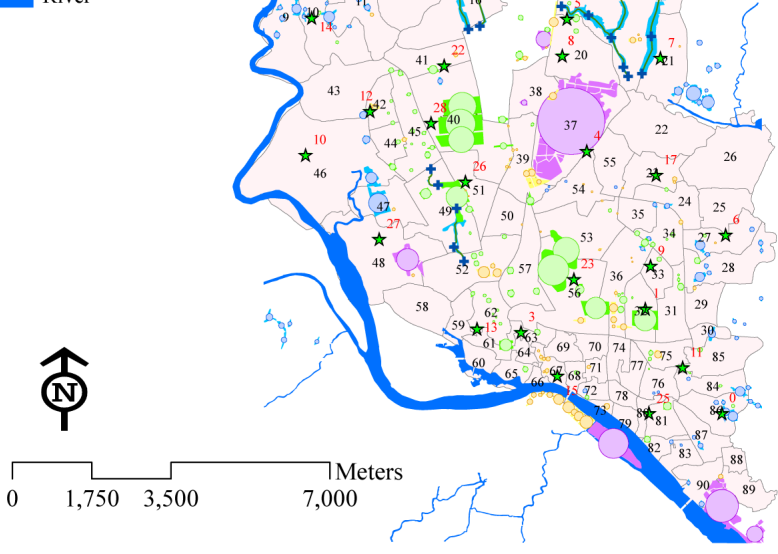

(a)

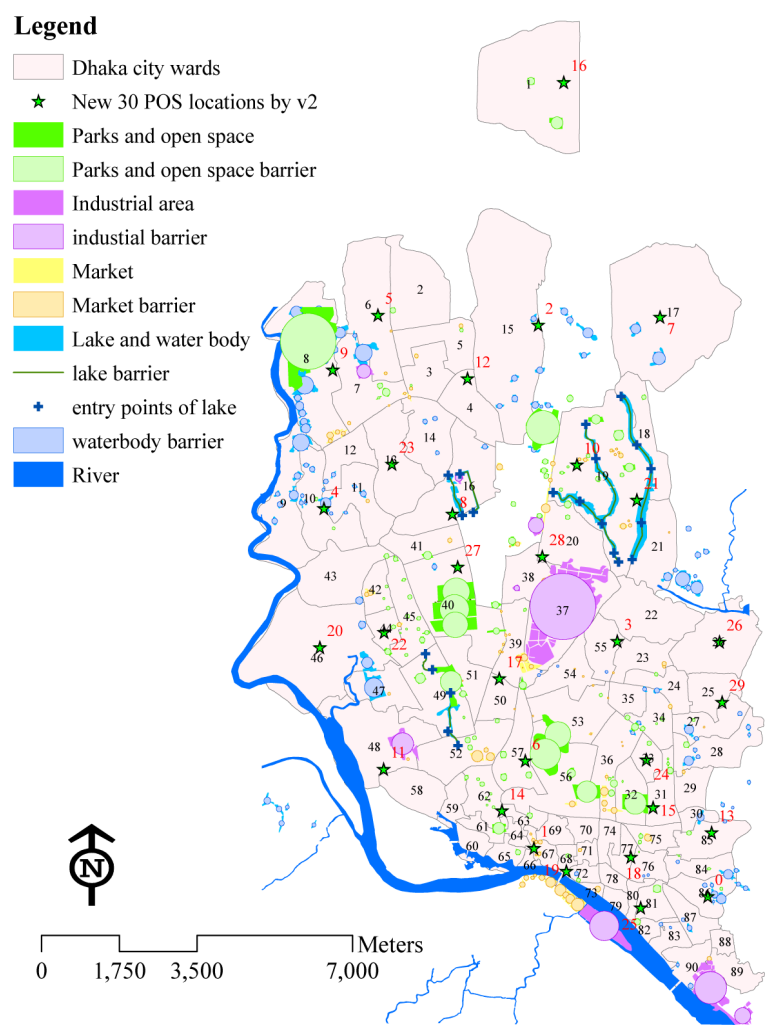

(b)

Legend

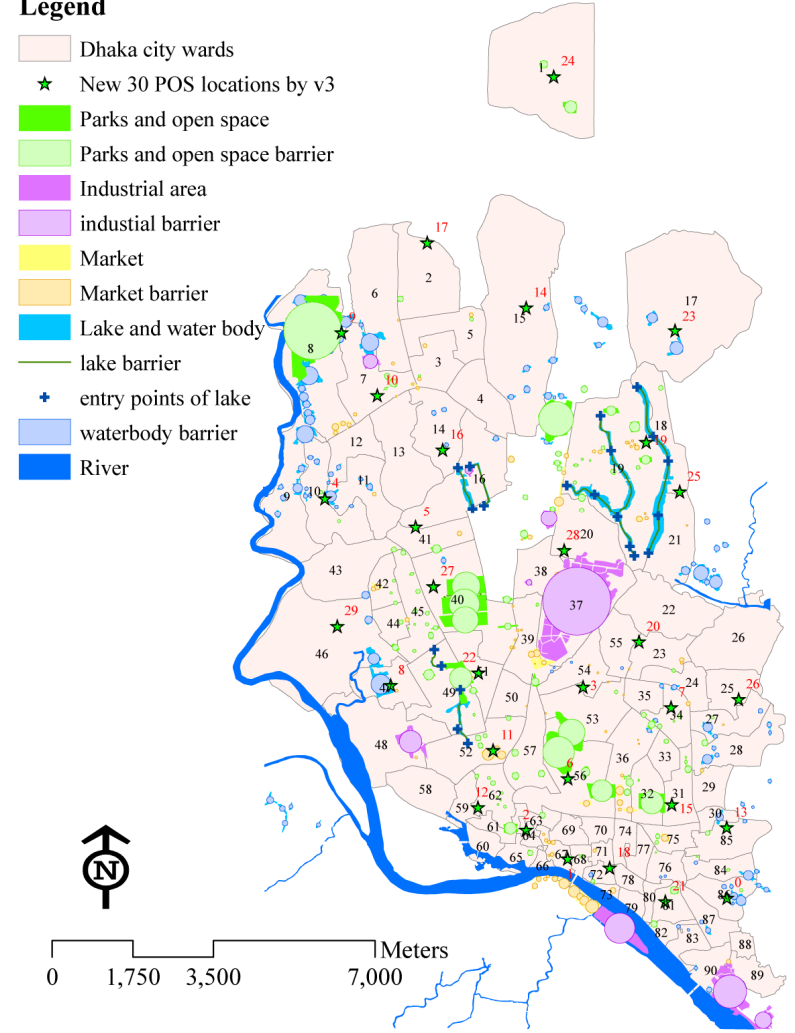

(c)

Figure 9.

New 30 POS locations found from the model by using: (a) $v_{1}=\left[\begin{array}{llll}0.41 & 0.38 & 0.20\end{array}\right]^{T}$, (b) $v_{2}=\left[\begin{array}{llll}0.30 & 0.44 & 0.26\end{array}\right]^{T}$ and (c) $v_{3}=\left[\begin{array}{llll}0.28 & 0.31 & 0.41\end{array}\right]^{T}$. 
spaces are beyond the scope of this paper.

However, to prevent the sitting (from simulations) of some new open spaces on the peripheries of barriers a pre-determined buffer region can be used. Indeed, considering a buffer zone from line barriers, the model has shown well distribution of open spaces exterior to the existing lake type barriers. For instance, locations of open spaces marked " 5 ”, “7” and " 19 " in Figure 9(a), location marked "21" in Figure 9(b) and location marked "9" in Figure 9(c) are completely outside to the peripheries of lake type barriers.

\section{Conclusion}

In this research, we developed an intelligent multi-objective continuous optimization model with a new approach for green urbanism in a city. This modelling approach seeks the optimum places for providing new parks and open spaces for greeneries throughout a city. Application of the model in Dhaka city has successfully demonstrated to provide optimal locations of additional POS. Adequate numbers of POS were found in environmentally degraded areas with air and noise pollution. In addition, the obtained locations of open spaces found near lakes and water bodies have shown to be planned in an integrated way (i.e. open spaces and lakes) by the city planners to bring a beautiful image and a better environment to rejuvenate city dwellers. As the developed powerful continuous optimization scheme in GA-based multi-objective model searches for a pool of nondominated Pareto optimal solutions, city planners can choose an alternative solution which is best suited for the prevailing land-use pattern in a city (if it is necessary by averting locations from city centre and developed residential areas choosing an appropriate solution from the pool). This model could equally be applicable in any city for providing optimum locations of POS. Following our approach, a well-planned urban greening can thus be realized to maintain a healthy sustainable city. However, the scope of the present study is currently limited to site the optimal locations of POS (especially for the purpose of urban greeneries), in future study it would be an interesting research aspect to incorporate wetlands and water bodies to find out optimum locations for all ecological reserves.

\section{Acknowledgements}

This research was conducted under Japanese Government Scholarship funding. We also acknowledge the funding from Hori Information Science Promotion Foundation, Japan.

\section{REFERENCES}

Azad, A., \& Kitada, T. (1998). Characteristics of the air pollution in the city of dhaka, bangladesh in winter. Atmospheric Environment, 32, 1991-2005. http://dx.doi.org/10.1016/S1352-2310(97)00508-6

BenDor, T., Westervelt, J., Song, Y., \& Sexton, J. (2013). Modeling park development through regional land use change simulation. Land Use Policy, 30, 1-12.

http://dx.doi.org/10.1016/j.landusepol.2012.01.012

Borrego, C., Martins, H., Tchepel, O., Salmim, L., Monteiro, A., \& Miranda, A. I. (2006). How urban structure can affect city sustainability from an air quality perspective. Environmental Modelling \& Software, 21, 461-467. http://dx.doi.org/10.1016/j.envsoft.2004.07.009

Camm, J., Norman, S., Polasky, S., \& Solow, A. (2002). Nature reserve site selection to maximize expected species covered. Operations Research, 50, 946-955.

Chiesura, A. (2004). The role of urban parks for the sustainable city.
Landscape Urban Plan, 68, 129-138.

http://dx.doi.org/10.1016/j.landurbplan.2003.08.003

Choumert, J. (2010). An empirical investigation of public choices for green spaces. Land Use Policy, 27, 1123-1131.

Drechsler, M., \& Wätzold, F. (2001). The importance of economic cost in the development of guidelines for spatial conservation management. Biological Conservation, 97, 51-59. http://dx.doi.org/10.1016/S0006-3207(00)00099-9

Egger, S. (2006). Determining a sustainable city model. Environmental Modelling \& Software, 21, 1235-1246.

http://dx.doi.org/10.1016/j.envsoft.2005.04.012

Fonseca, C. M., \& Fleming, P. J. (1991). An overview of evolutionary algorithms in multiobjective optimization. Evolutionary Computation, 3, 1-16. http://dx.doi.org/10.1162/evco.1995.3.1.1

Gobster, P. H. (1998). Urban parks as green walls or green magnets? interracial relations in neighborhood boundary parks. Landscape Urban Plan, 41, 43-55.

http://dx.doi.org/10.1016/S0169-2046(98)00045-0

Katz, N., \& Cooper, L. (1981). Facility location in the presence of forbidden regions, i: Formulation and the case of euclidean distance with one forbidden circle. European Journal of Operational Research, 6, 166-173. http://dx.doi.org/10.1016/0377-2217(81)90203-4

Klamroth, K. (2004). Algebraic properties of location problems with one circular barrier. European Journal of Operational Research, 154, 20-35. http://dx.doi.org/10.1016/0377-2217(81)90203-4

Kong, F., Yinb, H., Nakagoshic, N., \& Zongb, Y. (2010). Urban green space network development for biodiversity conservation: Identification based on graph theory and gravity modeling. Landscape Urban Plan, 95, 16-27. http://dx.doi.org/10.1016/j.landurbplan.2009.11.001

Lam, K. C., Ng, S. L., Hui, W. C., \& Chan, P. K. (2005). Environmental quality of urban parks and open spaces in Hong Kong. Environmental Monitoring and Assessment, 111, 55-73.

http://dx.doi.org/10.1007/s10661-005-8039-2

McDonnell, M., Possingham, H., Ball, I., \& Cousins, E. (2002). Mathematical methods for spatially cohesive reserve design. Journal of Environmental Modeling and Assessment, 7, 107-114.

Morancho, A. B. (2003). A hedonic valuation of urban green areas. Landscape Urban Plan, 66, 35-41.

http://dx.doi.org/10.1016/S0169-2046(03)00093-8

Neema, M.N., Ohgai, A., \& Emanuel, L. (2008). Analyzing existing condition and location of open spaces in Dhaka city. Proceedings of 6th Int. Symposium on City Planning and Urban Management in Asian Countries, Jinju.

Neema, M. N., \& Ohgai, A. (2010). Multi-objective location modeling of urban parks and open spaces: Continuous optimization. Computers, Environment and Urban Systems, 34, 359-376. http://dx.doi.org/10.1016/j.compenvurbsys.2010.03.001

Neema, M. N., Maniruzzaman, K. M., \& Ohgai, A. (2011). New genetic algorithms based approaches to continuous p-median problem. Networks and Spatial Economics, 11, 83-99. http://dx.doi.org/10.1007/s11067-008-9084-5

Neema, M.N., Maniruzzaman, K. M., \& Ohgai, A. (2013). Green urbanism incorporating greenery-based conceptual model towards attaining a sustainable healthy livable environment-Dhaka City's perspective. Current Urban Studies, 1, 19-27. http://dx.doi.org/10.4236/cus.2013.13003

Neema, M.N., \& Ohgai, A. (2013). Multitype green-space modeling for urban planning using GA and GIS. Environment and Planning B: Planning and Design, 40, 447-473. http://dx.doi.org/10.1068/b38003

Nordh, H., Hartigb, T., Hagerhalla, C., \& Frya, G. (2009). Components of small urban parks that predict the possibility for restoration. Urban Forestry Urban Greening, 8, 225-235. http://dx.doi.org/10.1016/j.ufug.2009.06.003

Poggio, L., \& Vräcaj, B. (2009). A GIS-based human health risk assessment for urban green space planning-an example from grugliasco (Italy). Science of the Total Environment, 407, 5961-5970. http://dx.doi.org/10.1016/j.scitotenv.2009.08.026

Schipperijn, J., Stigsdotter, U., Randrup, T. B., \& Troelsen, J. (2010). Influences on the use of urban green space-a case study in odense, denmark. Urban Forestry \& Urban Greening, 9, 25-32. http://dx.doi.org/10.1016/j.ufug.2009.09.002

Sdnp, B. (2005). Green Cities Plan for the Planet (Digital Publication). 


\section{N. NEEMA ET AL.}

Dhaka: World Enviornment Day.

Szeremeta, B., Henrique, P., \& Zannin, T. (2009). Analysis and evaluation of soundscapes in public parks through interviews and measurement of noise. Science of the Total Environment, 407, 6143-6149. http://dx.doi.org/10.1016/j.scitotenv.2009.08.039

Uddin, N. (2005). The relationship between Urban Forestry and Poverty Alleviation-Dhaka as a case study. Master Degree Project, Alnarp: Swedish University of Agricultural Sciences,.
Uy, P., \& Nakagoshi, N. (2008). Application of land suitability analysis and landscape ecology to urban green space planning in Hanoi, Vietnam. Urban Forestry Urban Greening, 7, 25-40.

http://dx.doi.org/10.1016/j.ufug.2007.09.002

Zhang, X., \& Armstrong, M. P. (2008). Genetic algorithms and the corridor location problem: multiple objectives and alternative solutions. Environment and Planning B, 35, 148-168.

http://dx.doi.org/10.1068/b32167 